\title{
PERFILES TURÍSTICOS EN FUNCIÓN DE LAS MOTIVACIONES PARA VIAJAR
}

\author{
Miguel Ángel Beltrán-Bueno \\ María Concepción Parra-Meroño \\ Universidad Católica San Antonio de Murcia
}

\section{RESUMEN}

El objetivo del presente artículo es la obtención de clusters o perfiles turísticos en función de las motivaciones turísticas, siguiendo el modelo de Crompton. Se ha realizado una encuesta a 859 personas a las que se preguntó por sus motivaciones a la hora de viajar, intención de viajar, búsqueda de información, percepción de la imagen de destino, forma de tomar las decisiones, cantidad de viajes, duración y gasto realizado y características sociodemográficas. Mediante un análisis de conglomerados se han obtenido cuatro perfiles turísticos: racionales, antropológicos, emocionales y hedonistas.

Palabras clave: motivaciones turísticas, perfiles turísticos, características del viajero, imagen de destinos turísticos.

\section{Tourist profiles based on the motivations}

\section{ABSTRACT}

The aim of this paper is to obtain clusters or tourist profiles based on tourist motivations, according to the model of Crompton. Data were collected from a sample of 859 individuals conducting a survey about travel motivations, intention of travelling, search for information, perception of destination image, way of taking decisions, number of trips, duration of stay and visitor expenditure and demographic and socio-economic characteristics. After doing a conglomerate analysis, four tourist profiles have been identified: rational, anthropological, emotional and hedonistic.

Keywords: tourist motivations, tourist profiles, traveler characteristics, image destinations.

Recibido: 15 de octubre de 2015

Devuelto para su revisión: 4 de mayo de 2016

Aceptado: 1 de junio de 2016

Facultad de Ciencias Jurídicas y de la Empresa. Universidad Católica San Antonio de Murcia. Avenida Jerónimos, 135.30107 Guadalupe MURCIA (España).E-mail: mabeltran@ucam.edu, mcparra@ucam.edu 


\section{INTRODUCCIÓN}

El sector turístico actual se caracteriza por cuatro aspectos básicos, esto es, un entorno muy competitivo y global, un elevado nivel tecnológico, unos clientes muy sofisticados y, en el caso de España, una situación de crisis económica con márgenes de beneficios y rentabilidades muy bajos. Esta última característica es coyuntural, sin embargo, las tres primeras son estructurales e, incluso, es de prever que su importancia sea mayor en un futuro próximo. A pesar de esta complejidad, es un sector clave que atrae inversiones e incorpora constantemente nuevas empresas, por lo que es necesario comprender muy bien cómo funciona el sistema turístico (Jafari, 1994; Cueva, 1998; entre otros), y, dentro de este, la demanda, o sea los turistas, ya que sin turistas el sistema turístico no tiene ninguna base empírica (Leiper, 1993).

Los turistas desarrollan su comportamiento en función de muchos factores que determinan dónde prefieren viajar, qué los motiva a viajar, cómo se organizan y buscan información, cuántas veces hacen turismo al año, cuánto tiempo duran los viajes, etc. Además, estas conductas o comportamientos sirven para segmentar el mercado turístico y trazar perfiles turísticos o grupos de turistas (actuales o potenciales) que comparten características que los diferencian del resto de segmentos. Precisamente para la obtención de estos perfiles o segmentos, los expertos en marketing utilizan los llamados criterios de segmentación (Kyzer, 1995) que pueden ser muy diversos (demográficos, socioeconómicos, motivacionales, psicográficos, entre otros). En las investigaciones sobre turismo se han realizado importantes esfuerzos con la finalidad de segmentar los distintos submercados del sector, siendo especialmente útiles para el marketing, tanto en su vertiente académica como empresarial (Ferreira, 2011; Molina, Gómez y Esteban, 2013; Rodríguez y Santana, 2014, entre otros).

Entre estos criterios se encuentran "las motivaciones", que explican el por qué los seres humanos se comportan de una determinada forma. Son el conjunto de estímulos conscientes o inconscientes, biológicos, psicológicos o sociales que impulsan a los individuos a actuar (Castaño, 2005), que en el caso del contexto de esta investigación, consiste en "viajar". El problema de estudiar las motivaciones es su complejidad ya que, por ejemplo, los individuos pueden viajar para descansar, para conocer otras culturas, para asistir a algún evento deportivo, entre muchos otros motivos. Sin embargo, en muchas ocasiones no tienen consciencia de la motivación por la cual se elige o se descarta un determinado destino y, en la mayoría de ellas, las motivaciones se presentan de forma conjunta, es decir, se viaja para descansar pero, al mismo tiempo, se visitan monumentos y se asiste a algún evento deportivo, o se viaja por motivos de negocios pero se realizan otras actividades gastronómicas, culturales, etc. (Krippendorf, 1987). No obstante, el estudio de las motivaciones permite entender mejor las expectativas, necesidades y objetivos de los turistas y es un elemento fundamental para diseñar estrategias de marketing y ayudar al posicionamiento y competitividad de los agentes implicados en la actividad turística (Pons, Morales y Díaz, 2007).

Así, el objetivo principal de esta investigación es la obtención de clusters o perfiles turísticos en función de las motivaciones turísticas, siguiendo el modelo teórico de Crompton (1979), que muchos otros autores han utilizado como base para sus investigaciones tanto teóricas como empíricas. 


\section{LAS MOTIVACIONES TURÍSTICAS}

El estudio de las motivaciones está relacionado con las necesidades y los deseos, sin embargo, se trata de conceptos diferentes y secuenciales, en el sentido de que las motivaciones están en un nivel intermedio entre las necesidades y los deseos (Schiffman y Kanuk, 2005). En primer lugar, todos los individuos tienen necesidades que son el resultado de carencias fisiológicas y mentales (Sandhusen, 2002), asociadas con la supervivencia y el bienestar social del ser humano, respectivamente. Las necesidades son una sensación de carencia de algo, un estado fisiológico o psicológico, que es común a todos los seres humanos y que es independiente de los factores étnicos y culturales (Schiffman y Kanuk, 2005). Tal y como advierte Quintanilla (2002) la necesidad tiene dos componentes: (1) la sensación de carencia de algo esencial para la supervivencia, y (2) la sensación de privación que es un aspecto subjetivo.

En el ámbito turístico, las motivaciones son las fuerzas socio-psicológicas que predisponen a los individuos a elegir y participar en una actividad turística (Iso-Ahola, 1982), y la literatura al respecto las considera elementos fundamentales en los modelos de elección de un destino turístico (Uysal y Hagan, 1993) y en los de formación de la imagen de destino (Stabler, 1990; Um y Crompton, 1990; Baloglu y McCleary, 1999). Mayo y Jarvis (1981) indican que las motivaciones de los turistas condicionan las imágenes de los destinos, y esto tiene lugar tanto de forma consciente como inconsciente (Moutinho, 1997). Además, como señala Pearce (1982) la motivación es un factor dinámico que cambia en función de la experiencia, el status y la edad. Por ello, se puede considerar a las motivaciones como el concepto central en la comprensión del comportamiento del turista (Castaño, 2005). Para Moutinho (1987) y Esteban (1996) las motivaciones constituyen uno de los factores clave que hay que identificar para comprender el comportamiento del turista y su decisión de compra.

En el ámbito de la psicología social "las motivaciones" explican el por qué los seres humanos se comportan de una determinada manera y son el conjunto de estímulos conscientes o inconscientes, biológicos, psicológicos o sociales que impulsan la acción hacia un determinado objetivo (Castaño, 2005). En la terminología turística, las motivaciones se consideran como las causas que empujan a los turistas a realizar determinadas acciones o a emprender un viaje, es decir, son personales, por lo que encierran un carácter subjetivo difícil de medir.

Por ello, la motivación es la fuerza de impulso que influye decisivamente en el conjunto de las acciones humanas incluido el comportamiento turístico (Crompton, 1979; Iso-Ahola, 1982; Ryan, 1997; Castaño, Moreno y Crego, 2006). Para Uysal y Hagan (1993) es el factor más relevante para entender el comportamiento del turista y, para muchos autores, es determinante en la formación de la imagen turística (Gunn, 1972; Um y Crompton, 1990, entre otros). No obstante, hay que advertir que un estudio completo sobre el comportamiento del turista debe contemplar otras variables causantes (aprendizaje, experiencia, clase social, grupos sociales, familia, cultura, percepciones, etc.) y, además, la motivación es multidimensional, es decir, el turista canaliza su comportamiento en función de una combinación de motivaciones (Prat, 2014a). También hay que indicar que es muy complejo determinar con exactitud cuáles son las motivaciones que influyen en la práctica turística; algunas son 
muy evidentes, como las motivaciones culturales, pero otras no lo son, permanecen ocultas, es decir, no se tiene consciencia de la motivación por la cual se elige o se descarta un determinado destino (Krippendorf, 1987). No obstante, el estudio de las motivaciones permite entender mejor las expectativas, necesidades y objetivos de los turistas y es un elemento fundamental para la creación de "productos vacacionales a medida" que permitan satisfacer mejor sus necesidades (Pons, Morales y Díaz, 2007, p. 94).

La motivación turística se concibe, en un principio, como un aspecto relacionado con las capacidades cognitivas de los individuos, que se manifiesta, según Dann (1977), a través de la necesidad de escape y de búsqueda. La necesidad de escape depende del estrés, la cotidianidad y la rutina, y la necesidad de búsqueda impele al turista a visitar lugares históricos, museos, etc.

A partir de esta aportación, casi todas las investigaciones que analizan en profundidad la relevancia de las motivaciones en el turismo citan a Crompton (1979) como el precursor del estudio de las motivaciones turísticas. Así, este autor distingue dos tipos de motivaciones: socio-psicológicas y culturales. Las primeras no están relacionadas con el destino sino con la necesidad de escapar de la rutina diaria, el estrés, la alienación, etc. (factores push), mientras que las segundas están relacionadas con el destino, y pueden ser muy diversas: el clima, la naturaleza, los monumentos y museos, etc. (factores pull), es decir, los factores empuje (push) son aquellos que inducen a alguien a tomar la decisión de emprender un viaje, mientras que los de arrastre (pull) son los responsables de la elección del destino (Uysal y Jurowski, 1994; Cha, McCleary y Uysal, 1995; Castaño, 2005). En la misma línea, Gnoth (1997) distingue entre motivos y motivaciones para denominar a los factores push y pull respectivamente. Así, los motivos implican que el turista tiene un impulso, una necesidad y actúa en consecuencia, y las motivaciones son el resultado de interacciones específicas de la persona y la situación y son procesos y efectos con mayor grado de definición y adopción (Gnoth, 1999: 248). Por ello, este autor distingue dos grandes grupos de investigaciones: (1) las que prestan especial atención a los motivos o factores push, que guardan relación con las necesidades de escape $y$, (2) las que inciden en las motivaciones o factores pull relacionados con la necesidad de búsqueda. Para algunos autores (Dann, 1977; Crompton, 1979), son los factores pull (culturales) los que mejor explican el comportamiento de los turistas. No obstante, tal y como advierten el propio Crompton (1979) y Krippendorf (1987), en el sector turístico es conveniente prestar mayor atención a los factores push (psico-sociológicos) en lo que a la elaboración de productos y el diseño de estrategias de promoción se refiere.

Así pues, son muchas las investigaciones que han utilizado las motivaciones como criterios subjetivos y específicos de segmentación, al estar relacionadas sobre todo con el componente afectivo de la imagen del destino turístico, es decir, con los beneficios buscados (Dann, 1996; Gartner, 1993; Walmsley y Jenkins, 1993; Baloglu, 1999; FuentesMoraleda, Muñoz-Mazón y Rodríguez-Izquierdo, 2016). La revisión de la literatura existente sobre la influencia de las motivaciones en el comportamiento del turista distingue aquellas investigaciones cuyo objetivo principal es crear un marco conceptual o modelo teórico relativo a las mismas (Gunn, 1972; Iso-Ahola, 1982; Pearce y Cantalbiano, 1982; Pearce, 1988, 1994; Haukeland, 1990; Cooper et al, 1998; Devesa, Laguna y Palacios, 2010a, 2010b; entre otros), de aquellas otras cuya finalidad es, por un lado, la obtención 
de perfiles turísticos utilizando las motivaciones como variable de segmentación y, por otro lado, la elaboración de estrategias de marketing apoyadas en dichas investigaciones (Gutiérrez y Bordás, 1993; Gnoth, 1997; Ryan y Glendon, 1998; McIntosh, Goeldner y Ritchie, 1999; De Borja, Casanovas y Bosch, 2002; Lee, Lee y Wicks, 2004; Severt, Wang, Chen y Breiter, 2007; Beh y Bruyere, 2007; Hsu, Cai y Wong, 2007; Devesa, Laguna y Palacios, 2010b; Prat, 2014b; entre otros).

Para el desarrollo de esta investigación se ha elegido el modelo de Crompton (1979) por dos motivos: en primer lugar, es el más referenciado y nombrado en la literatura al respecto y, en segundo lugar, se puede considerar un híbrido en lo que al tipo de investigación se refiere ya que plantea un doble objetivo (Castaño, 2005, p. 147); por un lado, la identificación de las motivaciones de los turistas y cómo influyen en la elección del destino turístico, y por otro, el desarrollo de un marco conceptual en relación con dichas motivaciones.

De acuerdo con este modelo y, en términos generales, los turistas viajan bien porque son empujados por motivos o variables internas o bien porque son atraídos por fuerzas externas del destino. Los factores de empuje (push factors) están relacionados con aspectos internos y emocionales, como el deseo de escapar, de descansar y relajarse, de aventura o de interacción social. Los factores de atracción (pull factors) están conectados con aspectos externos, cognitivos y de situación, como los atractivos del lugar, las infraestructuras recreativas o los escenarios culturales y naturales (Devesa, Laguna y Palacios, 2010a).

Hay que subrayar que el estudio llevado a cabo por Crompton no pretende distribuir las motivaciones en una población, ya que la muestra por él utilizada es muy pequeña y poco representativa para este fin, sino determinar o definir cuáles son las motivaciones que subsisten en el comportamiento del turista y, de acuerdo con las entrevistas realizadas, clasifica las motivaciones en dos grandes grupos: psicosociológicas y culturales, que a su vez se subdividen en otras motivaciones más específicas tal y como se puede observar en la Tabla 1.

\section{Tabla 1}

\section{CLASIFICACIÓN DE LAS MOTIVACIONES SEGÚN CROMPTON (1979)}

\begin{tabular}{|l|l|}
\hline \multicolumn{2}{|c|}{ Motivaciones psicosociológicas (factores push) } \\
\hline $\begin{array}{l}\text { Escape del } \\
\text { ambiente } \\
\text { cotidiano }\end{array}$ & $\begin{array}{l}\text { Consiste en un cambio temporal de ambiente diferente del lugar de residencia, } \\
\text { del hogar o del entorno laboral, es decir, el turista busca destinos diferentes de su } \\
\text { entorno habitual, tanto física como socialmente. }\end{array}$ \\
\hline $\begin{array}{l}\text { Exploración } \\
\text { y evaluación } \\
\text { del yo }\end{array}$ & $\begin{array}{l}\text { El viaje es una oportunidad para que el turista explore su propia identidad y para } \\
\text { el autodescubrimiento, así como para poner en práctica imágenes sobre sí mismo } \\
\text { y evaluarlas. }\end{array}$ \\
\hline Relajación & $\begin{array}{l}\text { Se refiere a la relajación mental que se deriva, por ejemplo, de la práctica de } \\
\text { hobbies e intereses que no se pueden desarrollar en la vida diaria, más que a la } \\
\text { relajación física. Esta última no siempre tiene lugar en las vacaciones, y algunos } \\
\text { de los entrevistados manifiestan haber llegado agotados tras las mismas. }\end{array}$ \\
\hline
\end{tabular}




\begin{tabular}{|c|c|}
\hline Prestigio & $\begin{array}{l}\text { Viajar supone un estilo de vida elevado, aunque con la democratización del acceso } \\
\text { a los medios de transporte y la generalización del turismo, el estatus asociado a } \\
\text { los viajes y al turismo es cada vez más difuso. }\end{array}$ \\
\hline Regresión & $\begin{array}{l}\text { Tiene lugar de dos maneras: (1) rompiendo con los roles y obligaciones cotidianas } \\
\text { que facilita un tipo de comportamiento más irracional, pueril o adolescente, y } \\
\text { (2) el turista también podría estar motivado por el deseo de retornar a una época } \\
\text { donde el estilo de vida era más simple, lejos de la complejidad e incertidumbre de } \\
\text { la actual sociedad, altamente tecnificada. }\end{array}$ \\
\hline $\begin{array}{l}\text { Mejora de las } \\
\text { relaciones de } \\
\text { parentesco }\end{array}$ & $\begin{array}{l}\text { Viajar es una buena oportunidad para reunirse con los miembros de la familia y } \\
\text { enriquecer sus relaciones. }\end{array}$ \\
\hline $\begin{array}{l}\text { Facilitación } \\
\text { de la } \\
\text { interacción } \\
\text { social }\end{array}$ & $\begin{array}{l}\text { Existen viajes más orientados a las personas que a los destinos. El período de } \\
\text { vacaciones es un tiempo propicio para conocer gente nueva y así intercambiar } \\
\text { opiniones, extender el número de contactos sociales o simplemente disfrutar } \\
\text { de gente que no es familiar. También puede existir el deseo de interaccionar } \\
\text { con la población local (anfitriones), si bien lograrlo reviste algunas dificultades } \\
\text { y muchos turistas acaban relacionándose con otros turistas. La cercanía en el } \\
\text { alojamiento y el participar en viajes organizados son factores que facilitan } \\
\text { la interacción social. Respecto de los viajes organizados, en ellos operan } \\
\text { una serie de elementos que propician este hecho: compartir experiencias, } \\
\text { proximidad física, existencia de individuos con intereses comunes en el grupo } \\
\text { o la incorporación al grupo de nuevos miembros que son introducidos por } \\
\text { individuos ya miembros. }\end{array}$ \\
\hline \multicolumn{2}{|r|}{ Motivaciones culturales (factores pull) } \\
\hline Novedad & $\begin{array}{l}\text { Se identifica con la curiosidad, la aventura, la búsqueda de lo nuevo y diferente y } \\
\text { sobre todo, en el sentido de vivir una nueva experiencia (con cierta independencia } \\
\text { del grado de conocimiento que se tenga del destino). No obstante, existen casos } \\
\text { en los que el viajero elige destinos que ya le son habituales, en concreto: (1) } \\
\text { pueden ser individuos que tratan de satisfacer necesidades psicosociológicas } \\
\text { más que motivos culturales, (2) adoptan una estrategia conservadora para reducir } \\
\text { el riesgo de que un destino poco familiar no resulte tan satisfactorio como el } \\
\text { ya conocido, y (3) pueden verse condicionados por el miedo a lo desconocido. } \\
\text { Así, en el turista podría darse una combinación de búsqueda de lo nuevo y de } \\
\text { estrategias de minimización de riesgos ante lo diferente. Entre estas estrategias } \\
\text { están los viajes organizados y la exposición gradual a situaciones novedosas, } \\
\text { tomando como punto de partida lo ya conocido. }\end{array}$ \\
\hline Educación & $\begin{array}{l}\text { El viaje es una forma de satisfacer necesidades educativas y de adquirir un bagaje } \\
\text { cultural, llegando a considerarse prácticamente una obligación moral de conocer } \\
\text { aquellos lugares que son particularmente accesibles a los sujetos. }\end{array}$ \\
\hline
\end{tabular}

Fuente: Crompton (1979) adaptado de Castaño (2005).

Cuadernos de Turismo, 39, (2017), 41-65 
Finalmente, en una revisión de la literatura sobre las motivaciones, hay que indicar que son muchos los autores que se han servido del modelo de Crompton para establecer diversos resultados, entre los que podemos citar a Park y Dawson (1998), Kozak (2002), Castaño, Moreno y Crego (2003), Kau y Lim (2005), Correia, Castaño, Moreno y Crego (2006), Sangpikul (2007), Devesa, Laguna y Palacios (2010a), Jönsson y Devonis (2008), Qiao, Chen, Guan y Kim (2008), Moreno, Beerli y Martín (2008), Sutithee (2009), Li y Cai (2009), Konu y Laukkanen (2009), Shi (2010), Devesa, Laguna y Palacios (2010b), Jensen (2011), Yousefi y Marzuki (2012), Dayour, (2013), Siaw y Ansah (2015), entre otros.

\section{METODOLOGÍA}

Los datos proceden del trabajo de campo que se realizó entre diciembre de 2013 y enero de 2014 por un equipo de encuestadores. En el mismo, se entrevistó a personas de ambos sexos, con edades comprendidas entre los 18 y los 65 años. El ámbito geográfico del estudio es representativo del sureste español, en concreto, la Región de Murcia y las provincias de Alicante y Almería, seleccionándose a los encuestados mediante un muestreo estratificado por cuotas de edad y sexo de acuerdo con los datos de población del INE para los residentes en las poblaciones mencionadas.

El cuestionario incluye escalas de intervalo tipo Likert de 11 puntos en las que 0 es la calificación más baja y 10 la más alta. También se han incluido preguntas categóricas y preguntas abiertas cuantitativas y discretas para medir diferentes variables.

A su vez, se ha dividido el cuestionario en cuatro bloques:

1. Bloque I. Para empezar, se pregunta al entrevistado que valore las diferentes opciones de viaje existentes (sol y playa, cultural, deporte, aventura, etc.) en el supuesto de tener dinero, tiempo y ganas de viajar a cualquier parte del mundo. También se pide que señale el motivo más frecuente para ir de turismo en los dos últimos años.

2. Bloque II. En este apartado se pregunta sobre las diferentes motivaciones del entrevistado a la hora de viajar. Para su medición y análisis se han utilizado 44 ítems, que se corresponden con las 9 motivaciones estudiadas previamente.

3. Bloque III. A continuación, se preguntan diversos aspectos relacionados con la forma de organizar el viaje, la búsqueda de información, la percepción del destino turístico, la cantidad de viajes anual, su duración y el gasto realizado.

4. Bloque IV. Por último, se preguntan cuestiones básicas para clasificar a los individuos en función de la edad, el sexo, la población y el nivel de estudios.

Para el tratamiento estadístico de los datos se ha utilizado software especializado, SPSS v.21 y EQS v6.1 para Windows.

La muestra final depurada se compone de 796 individuos, de los cuales 371 son hombres y 425 mujeres. En cuanto a la edad, los entrevistados la indicaron en años, agrupándose en tres tramos: de 18 y 28 años, de 29 a 48 años y de 49 a 65 años, quedando distribuidos como refleja la Tabla 2, y manteniéndose el equilibrio propuesto en este estudio. 
Tabla 2

DISTRIBUCIÓN DE LA MUESTRA POR SEXO Y EDAD

\begin{tabular}{llrrrr}
\hline & & \multicolumn{3}{c}{ Grupos de edad } & \multirow{2}{*}{ Total } \\
\cline { 3 - 5 } & & 18 a 28 & 29 a 48 & 49 a 65 & 371 \\
\hline \multirow{3}{*}{ Hombres } & Recuento & 129 & 126 & 116 & $46,6 \%$ \\
& \% del grupo edad & $46,2 \%$ & $46,8 \%$ & $46,8 \%$ & $46,6 \%$ \\
\hline \multirow{3}{*}{ Mujeres } & \% del total & $16,2 \%$ & $15,8 \%$ & $14,6 \%$ & 425 \\
& Recuento & 150 & 143 & 132 & $53,4 \%$ \\
& \% del grupo edad & $53,8 \%$ & $53,2 \%$ & $53,2 \%$ & $53,4 \%$ \\
\hline \multirow{3}{*}{ Total } & \% del total & $18,8 \%$ & $18,0 \%$ & $16,6 \%$ & 796 \\
& Recuento & 279 & 269 & 248 & $100,0 \%$ \\
& \% del grupo edad & $100,0 \%$ & $100,0 \%$ & $100,0 \%$ & $100,0 \%$ \\
\hline
\end{tabular}

En cuanto al nivel de estudios, la Tabla 3 muestra la distribución de la población encuestada dividida en cinco grupos. Se puede observar que el nivel de formación que más predomina es el de bachillerato o similar, si se tiene en cuenta que además de los que han indicado ese nivel, se deberían incluir también a los que estudian en la universidad, lo que supondría un porcentaje de 35,8 sobre el total. Por su parte el porcentaje de personas con estudios universitarios es bastante elevado, un 32,1\% afirman que poseen titulación universitaria.

Tabla 3

NIVEL DE ESTUDIOS DE LA MUESTRA

\begin{tabular}{lrrrr}
\hline & Frecuencia & $\%$ & $\%$ válido & $\%$ acumulado \\
\hline Básicos/primaria & 118 & 14,8 & 15,3 & 15,3 \\
Secundaria obligatoria & 119 & 14,9 & 15,5 & 30,8 \\
Bachillerato/similar & 199 & 25,0 & 25,9 & 56,7 \\
Estudia universidad & 86 & 10,8 & 11,2 & 67,9 \\
Titulado universitario & 247 & 31,0 & 32,1 & 100,0 \\
\hline Total válidos & 769 & 96,6 & 100,0 & \\
\hline
\end{tabular}

A partir de la propuesta de Crompton y de la revisión de diferentes estudios que han utilizado el modelo propuesto por este autor, se han establecido nueve factores motivacionales. Todos ellos se han medido en escala tipo Likert desde 0 (nada importante) a 10 (totalmente importante) y se muestran en la tabla 4. Además, fueron sometidos a la revisión de un grupo de expertos, y sus consideraciones fueron incluidas en la versión final del cuestionario. 
Tabla 4

ESCALAS DE MEDIDA DE LOS FACTORES MOTIVACIONALES

\begin{tabular}{|c|c|c|c|}
\hline Constructo & Código & Items - Descripción & Fuente \\
\hline $\begin{array}{l}\text { Exploración } \\
\text { y evaluación } \\
\text { del yo }\end{array}$ & $\begin{array}{l}{[\mathrm{C} 1]} \\
{[\mathrm{C} 2]} \\
{[\mathrm{C} 3]} \\
{[\mathrm{C} 4]} \\
{[\mathrm{C} 5]}\end{array}$ & $\begin{array}{l}\text { Experimentar/conocer nuevas culturas } \\
\text { Comprenderse mejor a sí mismo } \\
\text { Desarrollar sus habilidades y capacidades } \\
\text { Aprender cosas nuevas } \\
\text { Experimentar/vivir cosas diferentes }\end{array}$ & $\begin{array}{l}\text { Konu y Laukkanen } \\
\text { (2009) }\end{array}$ \\
\hline $\begin{array}{l}\text { Escapar del } \\
\text { ambiente } \\
\text { cotidiano }\end{array}$ & $\begin{array}{l}{[\mathrm{D} 1]} \\
{[\mathrm{D} 2]} \\
{[\mathrm{D} 3]} \\
{[\mathrm{D} 4]} \\
{[\mathrm{D} 5]}\end{array}$ & $\begin{array}{l}\text { Escapar del estrés del trabajo } \\
\text { Reducir la presión del día a día } \\
\text { Salir de la rutina diaria } \\
\text { Evadirse de los problemas cotidianos } \\
\text { Cambiar de aire y de rutinas }\end{array}$ & $\begin{array}{l}\text { Jensen }(2011) \\
\text { Devesa et al. } \\
\text { (2010a) }\end{array}$ \\
\hline Prestigio & $\begin{array}{l}{[\mathrm{E} 1]} \\
{[\mathrm{E} 2]} \\
{[\mathrm{E} 3]} \\
{[\mathrm{E} 4]} \\
{[\mathrm{E} 5]}\end{array}$ & $\begin{array}{l}\text { Contar a otros las experiencias del viaje } \\
\text { Ir a donde antes han ido los amigos } \\
\text { Ir a los lugares de moda } \\
\text { Ir a lugares confortables (con buenos hoteles y/o } \\
\text { restaurantes) } \\
\text { Ir a lugares de prestigio o que tengan fama }\end{array}$ & $\begin{array}{l}\text { Sangpikul (2007) } \\
\text { Moreno et al. } \\
\text { (2008) }\end{array}$ \\
\hline Relajación & $\begin{array}{l}{[\mathrm{F} 1]} \\
{[\mathrm{F} 2]} \\
{[\mathrm{F} 3]} \\
{[\mathrm{F} 4]} \\
{[\mathrm{F} 5]}\end{array}$ & $\begin{array}{l}\text { Aliviar el estrés y la tensión } \\
\text { Practicar actividades que le relajen } \\
\text { Disfrutar del buen tiempo } \\
\text { Simplemente descansar } \\
\text { Volver más calmado o tranquilo }\end{array}$ & $\begin{array}{l}\text { Moreno et al. } \\
\text { (2008) } \\
\text { Qiao, Chen, Guan y } \\
\text { Kim (2008) }\end{array}$ \\
\hline $\begin{array}{l}\text { Regresión o } \\
\text { nostalgia }\end{array}$ & $\begin{array}{l}{[\mathrm{G} 1]} \\
{[\mathrm{G} 2]} \\
{[\mathrm{G} 3]} \\
{[\mathrm{G} 4]} \\
{[\mathrm{G} 5]}\end{array}$ & $\begin{array}{l}\text { Anhelar situaciones del pasado } \\
\text { Mirar atrás y pensar en buenos tiempos vividos } \\
\text { Experimentar añoranza por recuerdos } \\
\text { Revivir el pasado o momentos del mismo } \\
\text { Volver a vivir cosas del pasado }\end{array}$ & $\begin{array}{l}\text { Pascal et al. (2002) } \\
\text { Konu y Laukkanen } \\
(2009)\end{array}$ \\
\hline $\begin{array}{l}\text { Mejorar las } \\
\text { relaciones } \\
\text { sociales }\end{array}$ & $\begin{array}{l}\mathrm{H} 1] \\
{[\mathrm{H} 2]} \\
{[\mathrm{H} 3]} \\
{[\mathrm{H} 4]} \\
{[\mathrm{H} 5]}\end{array}$ & $\begin{array}{l}\text { Tener vivencias con amigos/familiares } \\
\text { Pasar el tiempo con la familia/amigos } \\
\text { Confraternizar con familia/amigos } \\
\text { Mejorar sus relaciones familiares/amigos } \\
\text { Hacer escapadas con la pareja }\end{array}$ & Jensen (2011) \\
\hline $\begin{array}{l}\text { Facilitación de } \\
\text { la interacción } \\
\text { social }\end{array}$ & $\begin{array}{l}{[\mathrm{J} 1]} \\
{[\mathrm{J} 2]} \\
{[\mathrm{J} 3]} \\
{[\mathrm{J} 4]}\end{array}$ & $\begin{array}{l}\text { Hacer nuevas amistades } \\
\text { Relacionarse con los demás } \\
\text { Encontrar gente nueva y diferente } \\
\text { Tener o mejorar sus relaciones sociales }\end{array}$ & $\begin{array}{l}\text { Moreno et al. } \\
(2008) \\
\text { Park y Dawson } \\
(1998)\end{array}$ \\
\hline $\begin{array}{l}\text { Educación y } \\
\text { cultura }\end{array}$ & $\begin{array}{l}{[\mathrm{K} 1]} \\
{[\mathrm{K} 2]} \\
{[\mathrm{K} 3]} \\
{[\mathrm{K} 4]} \\
{[\mathrm{K} 5]}\end{array}$ & $\begin{array}{l}\text { Enriquecerse intelectualmente } \\
\text { Conocer otras culturas y formas de vida } \\
\text { Asistir a eventos culturales } \\
\text { Conocer lugares diferentes } \\
\text { Usar los viajes para aprender }\end{array}$ & $\begin{array}{l}\text { Moreno et al. } \\
(2008)\end{array}$ \\
\hline Novedad & $\begin{array}{l}\mathrm{L} 1] \\
{[\mathrm{L} 2]} \\
{[\mathrm{L} 3]} \\
{[\mathrm{L} 4]} \\
{[\mathrm{L} 5]}\end{array}$ & $\begin{array}{l}\text { Ver cosas nuevas y extraordinarias } \\
\text { Conocer diferentes grupos de personas } \\
\text { Experimentar novedades en la vida } \\
\text { Viajar a lugares con ambiente diferente } \\
\text { Sentir curiosidad por vivir cosas diferentes }\end{array}$ & $\begin{array}{l}\text { Yousefi y Marzuki } \\
\text { (2012) }\end{array}$ \\
\hline
\end{tabular}




\section{RESULTADOS}

\subsection{Validación de las escalas de medida de las motivaciones}

Antes de obtener los perfiles turísticos en función de las motivaciones, se ha llevado a cabo la validación de las escalas de medida de las motivaciones utilizando el programa EQS 6.1. Para la validación del instrumento de medida se ha comprobado su validez y fiabilidad.

Para comprobar el ajuste global del modelo, se ha realizado un Análisis Factorial Confirmatorio (AFC), procediendo a eliminar del modelo ${ }^{1}$ aquellos ítems cuyas cargas factoriales estandarizadas sean inferiores a 0,707 (Fornell y Larcker, 1981). Además, el residuo estandarizado medio absoluto del modelo de medida es 0,0536 y el error promedio de los elementos fuera de la diagonal es pequeño $(0,0561)$, lo que es un primer indicador de un buen ajuste, no existiendo en este sentido ningún ítem problemático.

Como se puede apreciar en la Tabla 5, el modelo presenta indicadores adecuados y dentro de lo recomendado por la literatura. Así, los Índices de Ajuste Incremental son superiores a 0,9 y el RMSEA es pequeño $(0,056)$. Además, la Chi-cuadrada normada es de 3,48 , inferior a 5 , lo que de acuerdo con Bollen (1989) es aceptable.

Tabla 5

AJUSTE GLOBAL DEL MODELO

\begin{tabular}{llr}
\hline & \multicolumn{1}{c}{ Indicador } & Resultados \\
\hline & Satorra-Bentler $\chi^{2}$ & $1.821,80$ \\
\cline { 2 - 3 } Medidas de ajuste global & Grados de libertad & 524 \\
\cline { 2 - 3 } & P-valor & 0,000 \\
\cline { 2 - 3 } & RMSEA & 0,056 \\
\cline { 2 - 3 } & IC (RMSEA) & 0,922 \\
\hline Medidas de ajuste & CFI & 0,923 \\
\cline { 2 - 3 } incremental & IFI & {$[0,053 ; 0,059]$} \\
\hline
\end{tabular}

Para comprobar la fiabilidad de las escalas de motivaciones (ver Tabla 6) se ha calculado el Alpha de Cronbach y la Fiabilidad Compuesta (FC), como medidas de la consistencia interna de las escalas, siendo en ambos casos superior a 0,7 , tal y como recomienda la literatura. Además, se ha comprobado también la Varianza Media Extraída (AVE), siendo ésta superior a 0,5 en todas las escalas (Fornell y Larcker, 1981).

1 Los datos que se presentan corresponden al modelo de medida final, una vez realizadas las correspondientes reespecificaciones, tras la eliminación de los ítems problemáticos. 
Tabla 6

INDICADORES DE FIABILIDAD DE LAS ESCALAS

\begin{tabular}{llccc}
\hline \multicolumn{1}{c}{ Factor } & \multicolumn{1}{c}{ Variables } & $\begin{array}{c}\text { Alpha de } \\
\text { Cronbach }\end{array}$ & $\begin{array}{c}\text { FC (Fiabilidad } \\
\text { Compuesta) }\end{array}$ & $\begin{array}{c}\text { AVE (Varianza } \\
\text { media extraída) }\end{array}$ \\
\hline [F1] Explorar & C1, C4, C5 & 0,75 & 0,77 & 0,53 \\
[F2] Escape & D1 a D5 & 0,90 & 0,91 & 0,67 \\
[F3] Prestigio & E1 a E3 & 0,80 & 0,81 & 0,58 \\
[F4] Relajación & F1, F2, F5 & 0,76 & 0,76 & 0,52 \\
[F5] Nostalgia & G1 a G5 & 0,96 & 0,96 & 0,83 \\
[F6] Relac. Pers. & H1 a H4 & 0,90 & 0,91 & 0,72 \\
[F7] Interac. Soc. & J1 a J4 & 0,93 & 0,93 & 0,77 \\
[F8] Cultura & K1, K2, K4, K5 & 0,83 & 0,83 & 0,55 \\
[F9] Novedad & L2 a L5 & 0,85 & 0,86 & 0,61 \\
\hline
\end{tabular}

Por otra parte, mediante el AFC se ha comprobado la validez convergente de las escalas motivacionales. Existe validez convergente cuando todos los ítems cargan significativamente en su correspondiente constructo y las cargas factoriales estandarizas alcanzan el valor mínimo de 0,707 o su promedio es de 0,7 o superior (Fornell y Larcker, 1981). Además, todas las cargas son significativas al $99 \%$.

En cuanto a la validez discriminante, se cumple si los ítems que miden un mismo concepto están relacionados entre sí y no con los ítems que miden otro concepto. Para su comprobación se ha utilizado el Test de la varianza extraída (Tabla 7) y el Test del intervalo de confianza (Fornell y Larcker, 1981).

Tabla 7

TEST DE LA VARIANZA EXTRAIDA

\begin{tabular}{|c|c|c|c|c|c|c|c|c|c|}
\hline & F1 & F2 & F3 & F4 & F5 & F6 & F7 & F8 & F9 \\
\hline $\mathrm{F} 1$ & 0,53 & 0,10 & 0,01 & 0,05 & 0,01 & 0,09 & 0,17 & 0,85 & 0,53 \\
\hline $\mathrm{F} 2$ & 0,31 & 0,67 & 0,04 & 0,78 & 0,02 & 0,12 & 0,04 & 0,08 & 0,09 \\
\hline F3 & 0,07 & 0,19 & 0,58 & 0,06 & 0,18 & 0,08 & 0,16 & 0,00 & 0,06 \\
\hline F4 & 0,23 & 0,88 & 0,25 & 0,52 & 0,04 & 0,13 & 0,05 & 0,03 & 0,06 \\
\hline F5 & 0,10 & 0,15 & 0,42 & 0,21 & 0,83 & 0,07 & 0,06 & 0,01 & 0,03 \\
\hline F6 & 0,29 & 0,35 & 0,27 & 0,37 & 0,27 & 0,72 & 0,18 & 0,08 & 0,12 \\
\hline F7 & 0,42 & 0,21 & 0,40 & 0,22 & 0,25 & 0,42 & 0,77 & 0,21 & 0,51 \\
\hline F8 & 0,92 & 0,29 & 0,06 & 0,18 & 0,10 & 0,28 & 0,42 & 0,55 & 0,53 \\
\hline F9 & 0,73 & 0,29 & 0,24 & 0,25 & 0,17 & 0,35 & 0,28 & 0,73 & 0,61 \\
\hline
\end{tabular}

En la diagonal aparece la AVE. Debajo de la diagonal, las correlaciones entre factores. Y sobre la diagonal, la varianza compartida (las correlaciones al cuadrado). 
Además, ninguno de los intervalos de confianza calculados contiene el 1, por lo que la validez discriminante también ha quedado confirmada (Sánchez-Pérez y Jiménez-Castillo, 2013; Vila, Küster y Aldás, 2000).

\subsection{Proceso para la obtención de cluster}

Para dar respuesta al objetivo de determinar los perfiles de los turistas en función de sus motivaciones se ha procedido, en primer lugar a construir las variables motivacionales siguiendo el modelo de Crompton (1979) sumando los ítems correspondientes a cada una. A continuación se ha realizado la tipificación de datos a valores comprendidos entre 0 y 1 para hacer comparables los datos de todas las variables motivacionales.

Para conseguir una clasificación o agrupamiento de los individuos en " $n$ ” grupos según el comportamiento de éstos en una serie de segmentos se ha utilizado el análisis de conglomerados (clusters). Esta técnica multivariante permite clasificar una población en un número pequeño de grupos mutuamente exclusivos y exhaustivos, sin tener en cuenta las posibles dependencias entre las variables, es decir, no pretende explicar estadísticamente ningún hecho concreto (Sánchez Cuenca, 1990).

Siguiendo a Aldenderfer y Blashfield (1984) el primer paso consiste en un estudio exhaustivo de las variables que se van incluir en dicho análisis, hecho ya realizado al comprobar las propiedades psicométricas de las diferentes escalas motivacionales, tal y como se ha explicado anteriormente.

En segundo lugar, y dado el gran número de casos existente, se ha utilizado el método K-medias para elaborar grupos similares y conocer sus características (Paz, 1989). Se trata de una técnica no jerárquica que comienza con una división del conjunto de los datos en "n" grupos configurados al azar para, a continuación, tratar de mejorar esta primera clasificación reasignando aquellos elementos que tienen una gran distancia al centroide del cluster al que pertenecen, posibilitando de esta forma la reducción de la distancia media entre cada elemento de un grupo y su centroide (Díaz de Rada, 1998). Se realizó el análisis y la clasificación con 3, 4 y 5 grupos, siendo la solución en 4 grupos la que mejor resultados dio, por lo que solo se presentan los resultados para este caso (Tabla 8).

\section{Tabla 8 \\ NÚMERO DE CASOS EN CADA CONGLOMERADO}

\begin{tabular}{lc|cc}
\hline \multicolumn{2}{c|}{ Núm. conglomerado } & $\mathrm{n}$ & Porcentaje \\
\hline \multirow{3}{*}{ Conglomerado } & 1 & 128 & $16 \%$ \\
& 2 & 214 & $27 \%$ \\
& 3 & 207 & $26 \%$ \\
\hline Válidos & 4 & 247 & $31 \%$ \\
\hline
\end{tabular}


La Tabla 9 contiene los centros iniciales, es decir, los valores que corresponden, en las nueve variables motivacionales utilizadas, a los casos que se han elegido como centros respectivos de los cuatro conglomerados solicitados.

\section{CENTROS INICIALES DE LOS CONGLOMERADOS}

\begin{tabular}{lcccc}
\hline \multirow{2}{*}{ Motivación } & \multicolumn{4}{c}{ Conglomerado } \\
\cline { 2 - 5 } Explorar & 1 & 2 & 3 & 4 \\
Escape & 0,82 & 0,86 & 1,00 & 0,18 \\
Prestigio & 0,47 & 0,42 & 1,00 & 0,66 \\
Relajación & 0,30 & 0,27 & 1,00 & 0,40 \\
Nostalgia & 0,63 & 0,07 & 1,00 & 1,00 \\
Relac. Pers. & 0,20 & 0,28 & 1,00 & 0,76 \\
Interac. Soc. & 0,03 & 1,00 & 1,00 & 0,94 \\
Cultura & 0,10 & 0,95 & 1,00 & 0,73 \\
Novedad & 0,63 & 0,91 & 1,00 & 0,37 \\
\hline
\end{tabular}

Una vez seleccionados los centros de los conglomerados, cada caso se asigna al conglomerado de cuyo centro se encuentra más próximo y comienza un proceso de ubicación iterativa de los centros hasta que ninguna reasignación de un caso a un nuevo cluster permita reducir más la distancia entre los individuos dentro de cada agrupamiento, ni aumentar la distancia entre los distintos clusters. El resultado final se presenta en la Tabla 10. La información mostrada se utiliza para conocer las características que definen a cada agrupamiento, considerando las puntuaciones medias de cada factor en los distintos clusters. En el conglomerado 1 los valores más elevados se corresponden con las motivaciones "relajación" y "exploración y evaluación del yo", en el conglomerado 2 con "exploración y evaluación del yo" y "educación y cultura", por su parte, en el conglomerado 3, son todas muy elevadas y, por último, en el conglomerado 4, las valoraciones más altas se corresponden con "relajación” y "mejorar las relaciones personales". Dada la importancia de la validación en el análisis de clusters, se analizarán en detalle las características de cada conglomerado después de comprobar la adecuación de la clasificación obtenida.

Una vez conocidos los centros de los conglomerados es interesante calcular el grado de diferencia entre ellos, considerando la distancia entre los centroides. A continuación, en la Tabla 11 se muestra dicha distancia en términos de distancia euclídea, de modo que los más parecidos son el conglomerado 4 con el resto y el que menos el 1 con el resto (Díaz De Rada, 1998). 
Tabla 10

CENTROS DE LOS CONGLOMERADOS FINALES

\begin{tabular}{lcccc}
\hline \multirow{2}{*}{ Motivación } & \multicolumn{4}{c}{ Conglomerado } \\
\cline { 2 - 5 } Explorar & 1 & 2 & 3 & 4 \\
Escape & 0,60 & 0,83 & 0,84 & 0,69 \\
Prestigio & 0,56 & 0,76 & 0,89 & 0,72 \\
Relajación & 0,35 & 0,37 & 0,66 & 0,56 \\
Nostalgia & 0,64 & 0,77 & 0,88 & 0,75 \\
Relac. Pers. & 0,26 & 0,21 & 0,66 & 0,56 \\
Interac Soc. & 0,54 & 0,74 & 0,87 & 0,73 \\
Cultura & 0,44 & 0,69 & 0,84 & 0,65 \\
Novedad & 0,56 & 0,83 & 0,85 & 0,68 \\
\hline
\end{tabular}

Tabla 11

DISTANCIAS ENTRE LOS CENTROS

DE LOS CONGLOMERADOS FINALES

\begin{tabular}{ccccc}
\hline Conglomerado & 1 & 2 & 3 & 4 \\
\hline 1 & 0,00 & 0,61 & 0,98 & 0,54 \\
2 & 0,61 & 0,00 & 0,60 & 0,47 \\
3 & 0,98 & 0,60 & 0,00 & 0,47 \\
4 & 0,54 & 0,47 & 0,47 & 0,00 \\
\hline
\end{tabular}

El último paso, siguiendo a Aldenderfer y Blashfield (1980), consiste en la validación de los resultados del análisis, para lo que se han utilizado dos técnicas: 1) el Análisis de la Varianza (ANOVA), con el objetivo de conocer las diferencias que presentan las variables en cada uno de los clusters y, 2) el análisis discriminante para saber el porcentaje de sujetos que están asignados correctamente. La utilización del análisis de la varianza se realizó para las tres agrupaciones estudiadas (tres, cuatro y cinco grupos) e indicó que la solución en cuatro conglomerados proporcionaba los mayores valores de F, lo que significa que ésta maximiza la distancia entre los clusters y minimiza la distancia entre los elementos de cada cluster. En la Tabla 12 se muestra el nombre de cada factor, la F de Fisher resultado de aplicar el Análisis de Varianza y la significación de ésta, siendo esta última columna la que nos indica que todos los factores producen diferencias significativas en la definición de los cuatro clusters. 
Tabla 12.

ANOVA DE LOS CONGLOMERADOS

\begin{tabular}{lcccrrr}
\hline & \multicolumn{2}{c}{ Conglomerado } & \multicolumn{2}{c}{ Error } & F & Sig. \\
\cline { 2 - 5 } & $\begin{array}{c}\text { Media } \\
\text { cuadrática }\end{array}$ & $\mathrm{gl}$ & $\begin{array}{c}\text { Media } \\
\text { cuadrática }\end{array}$ & $\mathrm{gl}$ & & \\
\hline Explorar & 2,27 & 3 & 0,02 & 792,00 & 130,35 & 0,00 \\
Escape & 2,95 & 3 & 0,03 & 792,00 & 95,22 & 0,00 \\
Prestigio & 4,14 & 3 & 0,03 & 792,00 & 125,38 & 0,00 \\
Relajación & 1,67 & 3 & 0,02 & 792,00 & 82,68 & 0,00 \\
Nostalgia & 9,58 & 3 & 0,03 & 792,00 & 335,15 & 0,00 \\
Relac. Pers. & 2,88 & 3 & 0,02 & 792,00 & 122,16 & 0,00 \\
Interac Soc. & 4,34 & 3 & 0,02 & 792,00 & 194,29 & 0,00 \\
Cultura & 3,03 & 3 & 0,02 & 792,00 & 174,01 & 0,00 \\
Novedad & 4,25 & 3 & 0,02 & 792,00 & 258,41 & 0,00 \\
\hline
\end{tabular}

En cuanto al análisis discriminante, se ha utilizado para conocer la forma en que un conjunto de individuos con determinadas características se puede clasificar en grupos previamente definidos, con el objeto de localizar las variables que mejor contribuyen a la clasificación de cada individuo (Paz, 1989).

De esta forma, en la Tabla 13 se muestra una tabla de contingencia entre los individuos pertenecientes a cada cluster y aquellos que están correctamente clasificados según este análisis. Por ejemplo, aunque en el cluster 1, del total de individuos (128) hay un individuo que el análisis lo coloca en el cluster 2 y tres en el cluster 4 y ocurre lo mismo en el resto de los grupos, lo relevante es que la clasificación en cuatro grupos es válida ya que el 97,4\% de los individuos está correctamente clasificado.

Tabla 13

MATRIZ RESUMEN DEL ANÁLISIS DISCRIMINANTE

\begin{tabular}{|c|c|c|c|c|c|c|c|}
\hline & & & \multicolumn{4}{|c|}{ Grupo de pertenencia pronosticado } & \multirow{2}{*}{ Total } \\
\hline & & & 1 & 2 & 3 & 4 & \\
\hline \multirow{8}{*}{ Original } & \multirow{4}{*}{ Recuento } & 1 & 124 & 1 & 0 & 3 & 128 \\
\hline & & 2 & 3 & 206 & 1 & 4 & 214 \\
\hline & & 3 & 0 & 0 & 206 & 1 & 207 \\
\hline & & 4 & 1 & 3 & 4 & 239 & 247 \\
\hline & \multirow{4}{*}{ Porcentaje } & 1 & 96,9 & 0,8 & 0,0 & 2,3 & 100,0 \\
\hline & & 2 & 1,4 & 96,3 & 0,5 & 1,9 & 100,0 \\
\hline & & 3 & 0,0 & 0,0 & 99,5 & 0,5 & 100,0 \\
\hline & & 4 & 0,4 & 1,2 & 1,6 & 96,8 & 100,0 \\
\hline
\end{tabular}

Clasificados correctamente el $97,4 \%$ de los casos agrupados originales. 


\subsection{Resultados generales de la muestra}

En cuanto a las motivaciones turísticas, para el conjunto de la muestra las motivaciones más valoradas son "relajación”, "exploración y evaluación del yo", "escape del ambiente cotidiano", "mejorar las relaciones personales" y "educación y cultura" con valores próximos a 0,75 . En el otro extremo, se encuentran "regresión y nostalgia" y "prestigio" con 0,44 y 0,50 , respectivamente (ver Tabla 14).

\section{Tabla 14
ESTADÍSTICOS DESCRIPTIVOS DE LAS MOTIVACIONES}

\begin{tabular}{lcc}
\hline \multicolumn{1}{c}{ Motivación $^{(1)}$} & Media & Desv. típ. \\
\hline Exploración y evaluación del yo & 0,75 & 0,16 \\
Escapar de la rutina & 0,75 & 0,20 \\
Prestigio & 0,50 & 0,22 \\
Relajación & 0,77 & 0,16 \\
Regresión o nostalgia & 0,44 & 0,25 \\
Mejora de las relaciones personales & 0,74 & 0,19 \\
Facilitación de la interacción social & 0,68 & 0,20 \\
Educación y cultura & 0,75 & 0,17 \\
Novedad & 0,70 & 0,18 \\
\hline (1)Valores normalizados $(0,1)$ & & \\
\hline
\end{tabular}

\subsection{Características de los clusters obtenidos}

Una vez obtenidos los cuatro clusters que mejor se ajustan a esta investigación, a continuación, se describen y definen los mismos en función de sus características más relevantes, y sobre todo, de aquellas que permiten una mejor diferenciación.

\section{a) Grupo 1. Racionales}

La característica más diferenciadora de este segmento es que se guían más por "lo que saben del mismo/sus creencias" con un $53,9 \%$ que por "las emociones que despiertan". Además, son razonablemente optimistas y emocionalmente estables.

Es el grupo menos numeroso, representa un $16,08 \%$ del total, se trata de individuos mayoritariamente hombres $(55,5 \%)$, de todas las edades y no son estudiantes, la mayoría tienen el bachiller o son titulados universitarios.

Aunque no discrimina especialmente, la motivación más importante es la relajación $(0,63)$ y lo que menos los motiva es la nostalgia $(0,26)$. El resto de las motivaciones ronda la media de 0,5 . 
En cuanto a la intención de viajar su motivo principal es para "descansar o escapar de la rutina sin más" con una puntuación de un 0,83 . Le sigue "pasar el tiempo con amigos" y "con la familia" con 0,80 y 0,76 , respectivamente.

El tipo de viaje mayormente realizado en los dos últimos años ha sido para "descansar o escapar de la rutina sin más" $(21,9 \%)$ y "disfrutar del sol y la playa" $(18,8 \%)$. A los individuos de este grupo no les atrae la cultura, la aventura, los deportes, la naturaleza, etc. En definitiva, no son dinámicos y viajan para pasar tiempo con la familia y los amigos.

En cuanto a la información que más les influye es aquella que procede de "la opinión de terceros (familiares y amigos)", ya que casi un 50\% del total de individuos de este grupo han elegido esta opción frente a la opción de dejarse influir por la literatura promocional, la opinión de expertos o los medios impersonales, como la radio o la televisión.

No realizan más de 2 o 3 viajes al año y la duración de los viajes es de 2 o 3 días como máximo, si bien la media es de 4,9 días. Por último, el gasto medio realizado no supera los 300 euros y, en muchos casos, el viaje lo pagaron otros $(18,4 \%)$.

\section{b) Grupo 2. Antropológicos}

Para estos individuos las motivaciones más importantes son "la cultura" $(0,83)$ y la "exploración y evaluación del yo" $(0,82)$. El "prestigio" y la "nostalgia" son las menos valoradas, apenas tienen importancia ( 0,37 y 0,21 , respectivamente). El resto de las motivaciones estudiadas también son importantes aunque en menor medida, con valores por encima de 0,70 .

Hay mucha similitud entre hombres y mujeres, aunque son mayoritariamente mujeres $(53,7 \%)$, de todas las edades y el segmento más significativo con un $43,6 \%$ es el de los titulados universitarios.

En cuanto a su intención de viajar su motivo principal es para "descansar o escapar de la rutina sin más" con una puntuación de un 0,80 . Le sigue "pasar el tiempo con amigos" y "con la familia" con 0,80 y 0,78 , respectivamente.

El tipo de viaje más realizado por este grupo ha sido para "descansar o escapar de la rutina sin más" $(31,8 \%)$ y "conocer otra cultura" $(18,2 \%)$. A los individuos de este grupo no les atrae especialmente "disfrutar del sol y de la playa" y "pasar tiempo en la naturaleza" y prácticamente nada el resto de opciones: la aventura, los deportes, aprender un idioma, etc. En definitiva, satisfacen sus necesidades de autorrealización con productos culturales, si bien, también viajan para pasar tiempo con la familia y los amigos.

Son individuos muy optimistas, emocionalmente estables y flexibles en lo referente a su personalidad turística y les gusta la novedad. Por otra parte, asumen el riesgo de equivocarse y no se presentan muy influenciables.

En cuanto a la información que más les influye es aquella que procede de "los medios impersonales" (folletos, catálogos, internet, etc.), ya que casi más de un 50\% del total de individuos de este grupo han elegido esta opción.

A la hora de decantarse por un destino turístico consideran más importante "las emociones que despierta" con un $67,3 \%$ que "el conocimiento y las creencias" que puedan tener sobre el mismo. 
Realizan aproximadamente 3 viajes al año como media y la duración de los viajes es corta, de 3 a 7 días como máximo, siendo la media de 6 días.

En cuanto al gasto medio, existe una gran dispersión. El porcentaje de individuos de este grupo que fueron invitados por terceros supone el 18,7\%.

\section{c) Grupo 3. Emocionales}

La mayoría de los individuos son mujeres (66,7\%), menores de 30 años con bachiller o título universitario. En lo referente al destino turístico, consideran que es mucho más importante "la/s emoción/es que despierta" con un 70,5\% que "lo que sabe del mismo/ sus creencias".

Estos individuos, en general, se sienten muy motivados para viajar, especialmente por "cultura" y "exploración y evaluación del yo personal" ( 0,83 y 0,82 , respectivamente).

En cuanto a su intención de viajar su motivo principal es para "descansar o escapar de la rutina sin más" con una puntuación de un 0,79 . Le sigue "pasar el tiempo con amigos" y "con la familia" con 0,78 y 0,75 , respectivamente.

El tipo de viaje más realizado por este grupo ha sido para "descansar o escapar de la rutina sin más" $(31,4 \%)$ y "pasar tiempo con la familia" o "amigos" (entre los dos 26,1\%). A continuación, han viajado para "disfrutar del sol y la playa" y "para conocer otra cultura" (12,1 y 10,6\%, respectivamente). El resto de las opciones son muy poco relevantes.

Son individuos muy optimistas, emocionalmente estables y flexibles en lo referente a su personalidad turística.

En cuanto a la información que más les influye es aquella que procede de "la opinión de familiares y amigos", ya que casi un $45 \%$ del total de individuos de este grupo han elegido esta opción.

Realizan una media de 2,93 viajes al cabo del año. Aproximadamente, un $80 \%$ de los individuos de este grupo realizan viajes inferiores a 7 días, siendo la media de 5,8 días.

En cuanto al gasto medio, existe una gran dispersión. El porcentaje de individuos de este grupo que fueron invitados por terceros asciende al 18,4\%.

\section{d) Grupo 4. Hedonistas}

Es el grupo más numeroso, representa un 31,03\% del total. Hay mucha similitud entre hombres y mujeres, aunque son mayoritariamente hombres $(53,4 \%)$, mayores de 48 años y la mayoría tienen el bachiller o son titulados universitarios.

En comparación con el resto de los grupos, conceden puntuaciones más bajas al conjunto de motivaciones, siendo sus motivaciones más relevantes "la relajación” y "escape de la rutina diaria" $(0,75$ y 0,72 , respectivamente). A diferencia de los grupos anteriores sí valoran la nostalgia $(0,57)$. Además, ningún ítem ha sobrepasado la valoración de 0,8 , si bien todas las opciones han sido valoradas por encima de 0,5, incluso "prestigio" y "nostalgia".

En cuanto a su intención de viajar su motivo principal es para "pasar el tiempo con amigos" con una puntuación de 0,80 . Le sigue "descansar o escapar de la rutina sin más" y "pasar tiempo con la familia" con 0,82 y 0,79 respectivamente. 
El tipo de viaje más realizado por este grupo ha sido para "descansar o escapar de la rutina sin más" $(20,02 \%)$ y "pasar tiempo con la familia" y "disfrutar del sol y la playa" (ambas con un 19\%).

En cuanto a la información que más les influye es aquella que procede de "la opinión de familiares y amigos", ya que casi un $48 \%$ del total de individuos de este grupo han elegido esta opción.

A la hora de decantarse por un destino turístico consideran más importante "las emociones que despierta" con un 55,09\% que "lo que sabe del mismo/sus creencias".

Realizan una media de 2,89 viajes al cabo del año. Aproximadamente, un $83 \%$ de los individuos de este grupo realizan viajes inferiores a 7 días, siendo la media de 5,5 días.

En cuanto al gasto medio, existe una gran dispersión, si bien, el 30\% de los individuos de este grupo gastaron, como media, unos 300 euros por viaje. El porcentaje de individuos de este grupo que fueron invitados por terceros supone el 19,4\%.

\section{CONCLUSIONES}

Para la realización del presente estudio se han validado las escalas de medida de las motivaciones propuestas por Crompton (1979). Los resultados han sido favorables en lo referente a la validez de contenido y la validez de los constructos (referida a la validez convergente y a la discriminante). También se ha comprobado la fiabilidad de las mencionadas escalas, siguiendo las recomendaciones de Sarabia y Cañadas (2013).

La validación de dichas escalas ha permitido, por una parte, evaluar la importancia de cada una de las motivaciones objeto de estudio y, por otro, la obtención de perfiles turísticos en función de las mismas. Así, en lo referente a las motivaciones, los individuos encuestados han valorado sobre todo y por igual, las siguientes: "relajación", "exploración y evaluación del yo", "escape del ambiente cotidiano", "mejorar las relaciones personales" y "educación y cultura". También se puede concluir que las motivaciones menos importantes son "regresión y nostalgia" y "prestigio".

Como se ha argumentado en la revisión teórica, nuestros resultados indican que las motivaciones son un buen criterio para segmentar el mercado turístico. Concretamente, el modelo validado permite obtener perfiles turísticos diferenciados; en nuestro caso, se han obtenido cuatro grupos de turistas, a los que hemos denominado racionales, antropológicos, emocionales y hedonistas. Cada uno de ellos tiene unas características y preferencias que los hace adoptar comportamientos similares a la hora de viajar y diferentes del resto de los grupos identificados.

1. Racionales. Se guían más por "lo que saben del destino turístico y sus creencias, que por las emociones que les despierta; viajan, sobre todo para "descansar o escapar de la rutina sin más" y pasar el tiempo con la familia y amigos" y "no les atrae la cultura, la aventura, los deportes o la naturaleza".

2. Antropológicos. Sus motivaciones principales para viajar son "la cultura" y la “exploración y evaluación del yo". A la hora de decantarse por un destino turístico consideran más importante "las emociones que despierta" que "el conocimiento previo y las creencias" que puedan tener sobre el mismo. 
3. Emocionales. Están muy motivados para viajar, especialmente por "exploración y evaluación del yo personal" y "cultura". En lo referente al destino turístico, consideran que es mucho más importante "las emociones que despierta" que "lo que sabe del mismo/sus creencias".

4. Hedonistas. A la hora de viajar lo que más les motiva es la "relajación" y el "escape de la rutina diaria", pero valoran bien otras motivaciones que los demás grupos lo hacen en menor medida como "nostalgia" y "prestigio. Además, se dejan influir por las emociones que les genera el destino turístico frente a la opinión de sus familiares y amigos.

Los resultados aquí obtenidos se pueden considerar en la línea de otras investigaciones previas, si bien, no se han encontrado estudios que hayan validado y medido todas las motivaciones descritas por Crompton, así, entre muchos otros, Kozak (2002) analiza las siguientes: 1) naturaleza, 2) escape, 3) interacción social y, 4) actividad física. Por su parte, Correia, Oom do Valle y Moço (2005) obtuvieron tres constructos motivadores de tipo push: (1) conocimiento/exploración, (2) escape/ocio y (3) interacción social; y, tres factores pull: (1) buenos servicios (playas, gastronomía, etc.), (2) atractivos principales y (3) características del medio ambiente. Sangpikul (2007) ha estudiado las siguientes motivaciones push y pull: 1) novedad, 2) descanso y relajación, 3) exploración y evaluación del yo, 4) culturales, 5) precio y programación de actividades, 6) compras, y 7) seguridad y limpieza. En la misma línea Jönsson y Devonis (2008) analizaron cómo influye la edad, género y nacionalidad en las siguientes motivaciones turísticas: 1) culturales, 2) hedonismo, 3) relajación, y 4) actividad física. Igualmente, Konu y Laukkanen (2009) describen las siguientes motivaciones; 1) exploración y evaluación del yo, 2) relajación y escape, 3) salud y actividad física, 4) regresión, 5) naturaleza, 6) prestigio, y 7) autonomía. Jensen (2011) destaca en su investigación 6 motivaciones; 1) exploración del yo personal, 2) escape-relax, 3) relaciones sociales con la familia/amigos, 4) prestigio, 5) búsqueda naturaleza y 6) culturales. Otros autores que se han basado en el modelo de Crompton son Yousefi, Maryam y Marzuki, Azizan (2012) que han analizado las siguientes motivaciones; 1) novedad, 2) exploración y evaluación del yo, 3) relajación, 4) medio ambiente y seguridad, 5) cultura y 6) servicios y atracciones

Igualmente, en la literatura española, son muchas las investigaciones que se han realizado para obtener perfiles turísticos (Cervantes, González y Muñiz, 1999; Castaño et al., 2006a; Devesa et al., 2010a; Escalona y Batista, 2011, entre otros), si bien, en ninguna se han estudiado las nueve motivaciones descritas en el modelo original de Crompton y la mayoría se centran en cuestiones relacionadas con destinos turísticos específicos. Se puede concluir, por tanto, que las nueve motivaciones propuestas son útiles para obtener perfiles turísticos con independencia de cuál sea el destino turístico concreto elegido.

En definitiva, las motivaciones propuestas por Crompton pueden seguir utilizándose en las investigaciones actuales, de hecho, así lo hacen un gran número de autores, y resultan acordes con la situación del mercado turístico y con los gustos y preferencias del consumidor turístico. Además, en el sector turístico conocer las motivaciones que impulsan a los turistas a viajar puede ser la clave de la atracción de los mismos por parte de los diferentes agentes dinamizadores del sector, ya sean turoperadores, agencias de viajes, hoteles, instituciones públicas, etc. 
No obstante, la clasificación de las motivaciones aquí utilizada no es la única posible; si bien nos ha permitido alcanzar los objetivos propuestos en esta investigación. Lo que sí es innegable, es el interés de la literatura científica en el estudio de las motivaciones de los turistas para comprender mejor el comportamiento de las personas a la hora de viajar, siendo este hecho la razón de ser del sistema turístico.

\section{BIBLIOGRAFÍA}

ALDENDERFER, M. y BLASHFIELD, R. (1984): Cluster Analysis. Beverly Hills, CA: Sage Press.

BALOGLU, S. (1999): «Path Analytic Model of Visitation Intention Involving Information Sources, Socio-Psychological Motivations, and Destination Image». Journal of Travel y Tourism Marketing, 8 (3), pp. 81-91.

BALOGLU, S. y MCCLEARY, K. (1999): «A Model, of Destinantion Image Formation». Annals of Tourism Research, 26 (4), pp. 868-897.

BEH, A. y BRUYERE, B. (2007): «Segmentation by visitor motivation in three Kenyan national reserves». Tourism Management, 28 (6), pp. 1464-1471.

BOLLEN, K. (1989): Structural equations with latent variables. NY: Wiley.

CASTAÑO, J. M. (2005): Psicología social de los viajes y del turismo. Madrid: Thomson.

CASTAÑO, J. M., MORENO, A. y CREGO, A. (2006): «Factores psicosociales y formación de imágenes en el turismo urbano: un estudio de caso sobre Madrid». PASOS. Revista de Turismo y Patrimonio Cultural, 4 (3), pp. 287-299.

CERVANTES, M., GONZÁLEZ, A., y MUÑÍZ, N. (1999): «La segmentación del mercado de los turistas de destinos de interior en la comercialización turística». Actas del $2^{\circ}$ Congreso de Turismo, Universidad y Empresa, pp. 77-96. Valencia: Tirant lo Blanch.

CHA, S., MCCLEARY, K. y UYSAL, M. (1995): «Travel motivations of Japanese Overseas Travellers: A Factor-cluster Segmentation Approach». Journal of Travel Research, 34 (1), pp. 33-39.

COOPER, C., FLETCHER, J., GILBERT, D. y WANHILL, S. (1998): Tourism: Principles and Practices. England: Adison Wesley: Longman (2nd ed.).

CROMPTON, J. L. (1979): «Motivations of pleasure vacations». Annals of Tourism Research, 6 (4), pp. 408-424.

CUENCA-SANCHEZ, J. (1990): «La Tipología». En E. ORTEGA, Manual de Investigación Comercial, pp. 524-539. Madrid: Pirámide.

CUEVA JIMÉNEZ, L. (1998): «La actividad turística española; una aproximación desde la dinámica de sistemas». Estudios Turísticos, 135, pp. 29-49.

DANN, G. (1977): «Anomie, ego-enhancement and tourism». Annals of Tourism Research, 4(4), pp. 184-194.

DANN, G. (1996): «Tourist images of a destination: an alternative analysis». En D. FESENMAIER, J. O'LEARY, y UYSAL, M. Recent Advances in Tourism Marketing Research. New York: The Haworth Press.

DAYOUR, F. (2013): «A study of backpackers» motivations in the Cape Coast-Elmina conurbation, Ghana». European Journal of Tourism, Hospitality and Recreation, 4 (3), pp. 69-94. 
DE BORJA, L., CASANOVAS, J.A. y BOSCH, R. (2002): El Consumidor Turístico. Madrid: Esic.

DEVESA, M., LAGUNA, M. y PALACIOS, A. (2010a): «Motivación, satisfacción y lealtad en el turismo: el caso de un destino de interior». Revista Electrónica de Motivación y Emoción. Monográfico de la Motivación del consumidor: Estudios experimentales. Vol. XIII, pp. 35-36.

DEVESA, M., LAGUNA, M. y PALACIOS, A. (2010b): «The role of motivation in visitor satisfaction: Empirical evidence in rural tourism». Tourism Management, 31, pp. 547-552.

DÍAZ DE RADA, V. (1998): «Diseño de tipologías de consumidores mediante la utilización conjunta del análisis cluster y otras técnicas multivariantes». Economía Agraria, 182 (Enero-Abril), pp. 75-104.

ESCALONA, R. y BATISTA, C. (2011): «Propuesta de perfiles de los turistas de Canadá en el destino Holguín». TURyDES. Revista de Investigación en Turismo y Desarrollo Local, Disponible en http://www.eumed.net/rev/turydes/09/ebbm.pdf.

ESTEBAN, Á. (1996): «El marketing turístico: la orientación de la actividad hacia el consumidor». En A. PEDREÑO MUÑOZ y V. MONFORT MIR, Introducción a la economía del turismo, pp. 247-273. Madrid: Editorial Cívitas.

FERREIRA, S.D. (2011): «Geo-segmentación y geo-posicionamiento en el análisis de las preferencias de los turistas: La geometría al servicio del marketing». Estudios y Perspectivas en Turismo. Volumen 2, pp. 842-854.

FORNELL, C. y LARCKER, D.R. (1981): «Evaluating structural equation models with unobservable variables and measurement error». Journal of Marketing Research, 18 (1), pp. 39-50.

FUENTES-MORALEDA, L., MUÑOZ-MAZÓN, A. y RODRÍGUEZ-IZQUIERDO, S. (2016): «El turismo solidario como instrumento de desarrollo: un estudio de caso para analizar las principales motivaciones de los turistas solidarios». Cuadernos de Turismo, 37, pp. 227-242.

GARTNER, W. (1993): «Image Formation Process». Journal of Travel y Tourism Marketing, 2 (2-3), pp. 191-215.

GNOTH, J. (1997): «Tourism motivation and expectation formation». Annals of Tourism Research, 24, pp. 283-304.

GUNN, C. (1972): Vacationscape: Designing Tourist Regions. Austin: University of Texas.

GUTIÉRREZ, C. y BORDAS, E. (1993): «Competitiveness of long haul tourist destinations». St. Gall (Suisse): AIEST (Vol. 35).

HAUKELAND, J.V. (1990): «Non-travellers: the flip side of motivations». Annals of Tourism Research, 17 (2), pp. 172-184.

HSU, C., CAI, L. y WONG, K. (2007): «A model of senior tourism motivations. Anecdotes from Beijing and Shanghai». Tourism Management, 28 (5), pp. 1262-1273.

ISO-AHOLA, S. (1982): «Toward a Social Psychological Theory of Tourism Motivation: A Rejoinde». Annals of Tourism Research, 9, pp. 256-262.

JAFARI, J. (1994: «La cientificación del turismo». Estudios y Perspectivas en Turismo, 3 (enero), pp. 7-36. 
JENSEN, J.M. (2011): «The relationships between socio-demographic variables, travel motivations and subsequent choice of vacation». Proceedings for the 2nd International Conference on Economics, Business and Management (pp. 37-44). Singapore: ICEBM (2011), Vol. 22.

KONU, H., y LAUKKANEN, T. (2009): Roles of Motivation Factors in Predicting Tourists' Intentions to Make Wellbeing Holidays. A Finnish Case. Disponible en: http:// www.duplication.net.au/ANZMAC09/ papers/ANZMAC2009-376.pdf

KRIPPENDORF, J. (1987): The Holiday Makers. Understanding the impact of leisure and travel. Londres: Butterworh Heinemann Oxford.

KYZER, O. (1995): «Narrow Focus Revea is Big Picture. Target Customers Using, Compiled Data on Demographics, Lifestyles and Behavior». Marketing Research: A magazine of Management and Applications, 7 (2), pp. 27-31.

LEE, C., LEE, Y. y WICKS, B. (2004): «Segmentation of festival motivation by nationality and satisfaction». Tourism Management, 25, pp. 61-70.

LEIPER, N. (1993): «Defining tourism and related concepts: tourims, market, industry and tourism system». En M. KHAN, M. OLSEN y T. VAR. Encyclopeida of Hospitality and Tourism, pp. 539-558. Nueva YorK: Van Nostrand Reinhold (VNR).

MAYO, E. y JARVIS, L. (1981): The Psychology of Leisure Travel. Boston: CBI Publishing Company.

MCINTOSH, R., GOELDNER, C. y RITCHIE, J.R.B. (1999): Turismo Planeación, Administración y Perspectiva. México: Limusa-Wiley.

MOLINA, A., GÓMEZ, M. y ESTEBAN, A. (2013): «Segmentación de la demanda turística: un análisis aplicado a un destino de turismo cultural». Papers de turisme, 53, pp. 1-17 Artículo.

MORENO, S., BEERLI, A. y MARTÍN SANTANA, J. (2008): «La concepción sistémica de la imagen del destino: una aproximación desde la imagen del alojamiento». Recuperado el 10 de Febrero de 2016, de www.esade.es/cedit2004/pdfs/41_ Moreno.pdf.

MOUTINHO, L. (1987): «Consumer Behaviour in Tourism». European Journal of Marketing, 21 (10), pp. 1-44.

PARK, C.I., y DAWSON, C. (1998): «An exploratory comparison of motivations and crowding norms between ethnic groups in downhill ski areas of New York state and Korea». En H. G. Vogelsong, Proceedings of the 1997 Northeastern Recreation Research Symposium (pp. 56-59). Northeastern Forest Experiment Station: Department of Agriculture, Forest Service.

PASCAL, V. J., SPROTT, D. E., y MUEHLING, D D. (2002): «The influence of evoked nostalgia on consumers responses to advertising: an exploratory study». Journal of Current Issues and Research in Advertising, 24 (1), pp. 39-49.

PAZ, M.D. (1989): «Análisis de Cluster». En G. VALLEJO, Análisis Multivariante aplicado a las ciencias del comportamiento, pp. 277-279. Oviedo: Universidad de Oviedo.

PEARCE, P. (1988): The Ulysses factor: Evaluation of visitors in tourist settings. Nueva York: Springer Verlag.

PEARCE, P. (1982): «Perceived changes in holiday destinations». Annals of Tourism Research, 9, pp. 145-164. 
PEARCE, P. y CANTALBIANO, N. (1982): «Gesture decoding and encoding in children: the effects of ethnicity, age and sex». Australian Journal of Psychology, 34, pp. 17-24.

PRAT, J.M. (2014a): «Las relaciones sociales y las motivaciones para asistir al festival de música de Peralada». Boletín de la Asociación de Geógrafos Españoles, 66, pp. 207-221.

PRAT, J M. (2014b): «Nuevos planteamientos y nuevas motivaciones en la elección de destinos de turismo cultural-urbano homosexual: el Festival Circuit de Barcelona». Cuadernos de Turismo, 33, pp. 311-334.

PONS, R., MORALES, L. y DÍAZ GONZÁLEZ, Y. (2007): «La imagen del destino y el comportamiento de compra del turista». Teoría y Praxis, 3, pp. 89-102.

QIAO, G. H., CHEN, N., GUAN, Y. Y., y KIM, S. C. (2008): «Study on chinese tourists» motivation and satisfaction to visit South Korea». International Journal of Tourism Sciences, 8 (1), pp. 17-38.

QUINTANILLA, I. (2002): Psicología del consumidor. Madrid: Prentice Hall.

RODRÍGUEZ, P. y SANTANA, M. (2014). «Consumo turístico y desigualdad social en España». Pasos: Revista de turismo y Patrimonio Cultural, 12 (1), 29-51.

RYAN, C. (1997): The Tourist Experience: a New introduction. Londres: Cassell. UK.

RYAN, C. y GLENDON, I. (1998): «Application of leisure motivation scale to tourism». Annals of Tourism Research, 25(1), pp. 169-184.

SANDHUSEN, R. (2002): Mercadotecnia. Mexico: Compañía Editorial Continental.

SANGPIKUL, A. (2007): «A factor-cluster analysis of tourist motivations: A case of U.S. senior travellers». Original Scientific Paper, 56(1), pp. 23-40.

SARABIA, F. J. y CAÑADAS, I. (2013): «Fiabilidad del instrumento de medida». En F. J. SARABIA, Métodos de investigación social y de la empresa, pp. 367-386. Madrid: Pirámide.

SCHIFFMAN, L. y KANUK, L. (2005): Comportamiento del Consumidor. México, D.F: Pearson Educación ( $8^{\mathrm{a}}$ edición).

SEVERT, D., WANG, Y, CHEN, P.J. y BREITER, D. (2007): «Examining the motivation, perceived performance, and behavioural intentions of convention attendees: Evidence from a regional conference». Tourism Management, 28, pp. 399-408.

SIAW, L. y ANSAH, J (2015): Tourist Destination Choice: Motivational factors among Social Science Students' at Knust. http://www.academia.edu/10832099/Tourist_Destination_Choice_Motivational_factors_among_Social_Science_Students_at_KNUST. Recuperado el 13 de marzo de 2015

STABLER, M. (1990): «The image of destination regions: theoretical and empirical aspects». En B. GOODALL, y G. ASHWORTH, Marketing in the Tourism Industry: The Promotion of Destination Regions, pp. 133-161. Londres: Routledge.

UM, S. y CROMPTON, J.L. (1990): «Attitude determinants in tourism destination choice». Annals of Tourism Research, pp. 17, 432-448.

UYSAL, M. y HAGAN, L. (1993): «Motivation of pleasure travel and tourism». En M. KHAN, M. OLSEN y VAR, T. Encyclopedia of Hospitality and Tourism, pp. 798-810. New York: Van Nostrand Reinhold. 
UYSAL, M. y JUROWSKI, C. (1994): «Testing the push and pull factors». Annals of Tourism Research, 21(4), pp. 844-846.

VILA, N. KÜSTER, I. y ALDÁS, J. (2000): Desarrollo y validación de escalas de medida en marketing. Valencia: Servei de Publicacions, Facultat de Ciències Econòmiques i Empresarials, Universitat de València.

WALMSLEY, D. y JENKINS, J. (1993): «Appraisive images of tourist areas: Application of personal construct». Australian Geographer, vol. 24 (2), pp. 1-13.

YOUSEFI, M. y MARZUKI, A. (2012): «Travel motivations and the influential factors: the case of Penang. Malaysia. Anatolia». International Journal of Tourism and Hospitality Research, 23 (2), pp. 169-176. 
